\title{
Periodic Orbits in the Photogravitational Restricted Problem When the Primaries Are Triaxial Rigid Bodies
}

\author{
Preeti Jain', Rajiv Aggarwal'2, Amit Mittal'1, Abdullah ${ }^{3}$ \\ ${ }^{1}$ Department of Mathematics, A.R.S.D. College, University of Delhi, New Delhi, India \\ ${ }^{2}$ Department of Mathematics, Sri Aurobindo College, University of Delhi, New Delhi, India \\ ${ }^{3}$ Department of Mathematics, College of Science in Zulfi, Majmaah University, Riyadh, KSA \\ Email: to.amitmittal@gmail.com,rajiv_agg1973@yahoo.com
}

Received 2 December 2015; accepted 26 March 2016; published 30 March 2016

Copyright (C) 2016 by authors and Scientific Research Publishing Inc.

This work is licensed under the Creative Commons Attribution International License (CC BY). http://creativecommons.org/licenses/by/4.0/

\section{(c) (i) Open Access}

\begin{abstract}
We have studied periodic orbits generated by Lagrangian solutions of the restricted three-body problem when both the primaries are triaxial rigid bodies and source of radiation pressure. We have determined periodic orbits for different values of $\mu, h, A_{1}, A_{1}^{\prime}, A_{2}, A_{2}^{\prime}, P$ and $P^{\prime}$ ( $h$ is energy constant; $\mu$ is mass ratio of the two primaries; $A_{1}, A_{1}^{\prime}, A_{2}$ and $A_{2}^{\prime}$ are parameters of triaxial rigid bodies and $P$ and $P^{\prime}$ are radiation parameters). These orbits have been determined by giving displacements along the tangent and normal at the mobile co-ordinates as defined in our papers (Mittal et al. [1]-[3]). These orbits have been drawn by using the predictor-corrector method. We have also studied the effect of triaxial bodies and source of radiation pressure on the periodic orbits by taking fixed value of $\mu$.
\end{abstract}

\section{Keywords}

Restricted Three-Body Problem, Periodic Orbits, Triaxial Rigid Body, Radiation Pressure

\section{Introduction}

This paper is the extension of our papers, Mittal et al. [1]-[3]. Charlier [4] and Plummer [5] studied the existence of two families of small periodic motions in the neighborhood of Lagrangian solutions in the plane circular restricted three-body problem, with different values of the parameter $\mu$. Riabov [6] investigated periodic motions analytically. Szebehely [7] has described the results on the periodic motions of circular restricted three-body problem. Deprit and Henrard [8] gave more results on periodic motions in their paper. Markeev and Sokolsky 
[9] worked on the small periodic motions generated by Lagrangian solutions for all values of $\mu$ and for small values of $h$ for which the conditions of holomorphic integral theorem are valid. Hadjidemetriou [10] has discussed the continuation of periodic orbits from the restricted to the general three-body problem. Karimov and Sokolsky [11] have studied the periodic motions generated by Lagrangian solutions of the circular restricted three-body problem by using mobile co-ordinates and by taking displacements along the tangent and the normal. Aggarwal et al. [12] have discussed the non-linear stability of the triangular libration point when both the primaries are radiated axe symmetric rigid bodies in the presence of third and forth order resonance. Abouelmagd et al. [13] have studied the periodic structure of the restricted three-body problem considering the effect of the zonal harmonics $J_{2}$ and $J_{4}$ for the more massive body. They showed that the triangular points in the restricted three-body problem have long or short periodic orbits in the range $0 \leq \mu<\mu_{c}$. Perdios et al. [14] have studied the equilibrium points and related periodic motions in the restricted three-body problem with angular velocity and radiation effects. Jain and Aggarwal [15] have studied the stability and existence of non-collinear libration points in restricted three-body problem with stokes drag effect when smaller primary is an oblate spheroid.

The celestial bodies are in general axis-symmetric bodies, so its shape should be taken into account as well. The replacement of mass point by rigid-body is quite important because of its wide applications. The re-entry of artificial satellite has shown the importance of periodic orbits.

That is why, we have thought of studying, in this paper, the periodic orbits generated by Lagrangian solutions of the restricted three-body problem when both the primaries are triaxial rigid bodies and source of radiation pressure. We determine the periodic orbits by giving displacements at the mobile co-ordinates along the tangent and normal. We have also determined family of periodic orbits by fixing $\mu$ (mass ratio of the two primaries) and changing the values of $A_{1}, A_{2}, A_{1}^{\prime}, A_{2}^{\prime}$ (parameters of the triaxial rigid bodies), $P$ and $P^{\prime}$ (the radiation parameters) and varying $h$ (energy constant). We have also studied the effect of triaxial parameters and the radiation pressure on the energy constant $(h)$.

Most of the authors have not taken into account the effect of the solar radiation pressure in the motion of the third body whereas we have taken both the primaries as radiating triaxial rigid bodies. Besides taking both the primaries as triaxial rigid bodies and the source of radiation, we have used mobile-coordinates and given the displacement along the normal and the tangent to the orbit which has wider applications in space dynamics. We have drawn the periodic orbits by using the predictor-corrector method which is given in detail in our papers [1]-[3].

\section{Equations of Motion}

Following the procedure of our papers [1]-[3], we consider three masses $m_{1}, m_{2}$ and $m_{3}$ with $m_{1} \geq m_{2}$ and bodies with masses $m_{1}$ and $m_{2}$ revolve with angular velocity $n$ (say) in circular orbits without rotation about the centre of mass $O$. Let there be an infinitesimal mass $m_{3}$ which is moving in the plane of motion of $m_{1}$ and $m_{2}$ and is being influenced by their motion but not influencing them. Let the line joining $m_{1}$ and $m_{2}$ be taken as $X$-axis and $O$ their centre of mass as origin. Let the line through $O$ and perpendicular to $O X$, and lying in the plane of motion of $m_{1}$ and $m_{2}$ being $Y$-axis. Let us consider a synodic system of coordinates $O(X Y Z)$, initially coincident with the inertial system $O(X Y Z)$, rotating with the angular velocity $n$ about $Z$-axis; (the $Z$-axis is coincident with $Z$-axis). We choose unit of mass such that $m_{1}+m_{2}=1$, the unit of distance $A B=1$ and unit of time is so chosen that $G=1$. Using the dimensionless variables, we find the Lagrangian function $L$ and the equations of motion of the infinitesimal mass in the restricted three-body problem when both the primaries are radiating triaxial rigid bodies in the synodic co-ordinate system (Figure 1).

Equations of motion with Lagrangian function $L$ are given by

$$
\frac{\mathrm{d}}{\mathrm{d} t}\left(\frac{\partial L}{\partial \dot{x}}\right)-\frac{\partial L}{\partial x}=0, \frac{\mathrm{d}}{\mathrm{d} t}\left(\frac{\partial L}{\partial \dot{y}}\right)-\frac{\partial L}{\partial y}=0,
$$

where

$$
\begin{aligned}
L= & \frac{1}{2}\left(\dot{x}^{2}+\dot{y}^{2}\right)+n(x \dot{y}-\dot{x} y)+\frac{n^{2}}{2}\left(x^{2}+y^{2}\right)+(1-\mu)\left(\frac{1}{r_{1}}+\frac{A_{1}}{2 r_{1}^{3}}+\frac{3 A_{2} y^{2}}{2 r_{1}^{5}}-\frac{P}{r_{1}}\right) \\
& +\mu\left(\frac{1}{r_{2}}+\frac{A_{1}^{\prime}}{2 r_{2}^{3}}+\frac{3 A_{2}^{\prime} y^{2}}{2 r_{2}^{5}}-\frac{P^{\prime}}{r_{2}}\right)+U,
\end{aligned}
$$




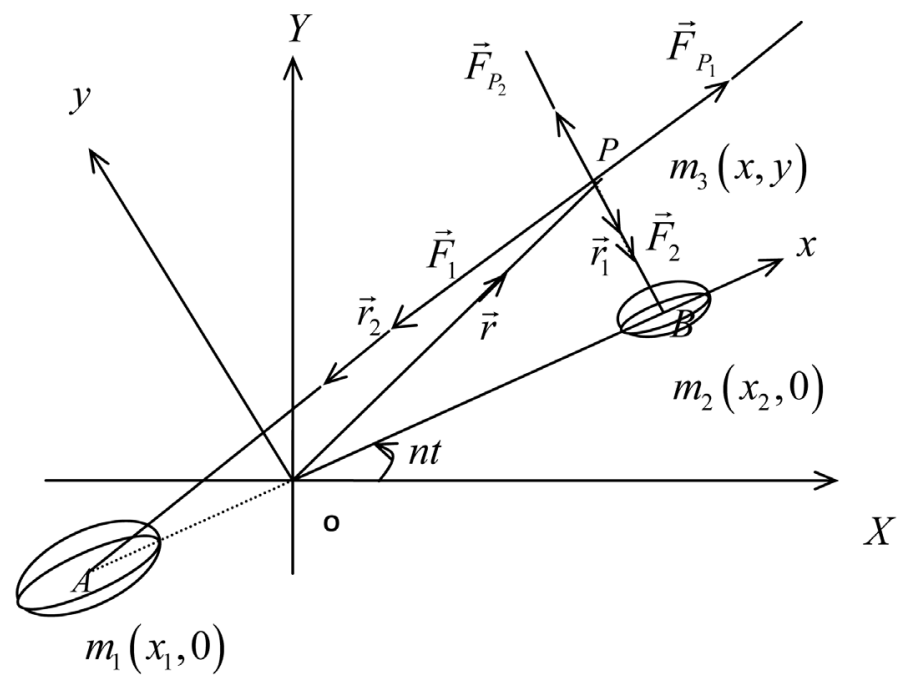

Figure 1. Configuration of the restricted three-body problem with masses $m_{1}$ and $m_{2}$ as radiating triaxial rigid bodies.

$(x, y)=$ the synodic rectangular dimensionless co-ordinates of the infinitesimal mass $m_{3}$.

Here we have assumed both the primaries, with masses $m_{1}$ and $m_{2}$ are radiating triaxial rigid bodies,

$n=$ mean motion $=1+\frac{3}{4} A_{1}+\frac{3}{4} A_{1}^{\prime} ; A_{1}$ and $A_{1}^{\prime}$ are the parameters of triaxial rigid bodies. It may be noted that $n$ is independent of $A_{2}$ and $A_{2}^{\prime}$ and $P$ and $P^{\prime}$ (the radiation parameters),

$$
\begin{gathered}
P=\frac{\text { Radiation pressure due to the bigger primary }}{\text { Gravitational force due to the bigger primary }} \\
P^{\prime}=\frac{\text { Radiation pressure due to the smaller primary }}{\text { Gravitational force due to the smaller primary }}, \\
A_{1}=\frac{2 a^{2}-b^{2}-c^{2}}{5 R^{2}}, A_{1}^{\prime}=\frac{2 a^{\prime 2}-b^{\prime 2}-c^{\prime 2}}{5 R^{2}}, \\
A_{2}=\frac{b^{2}-a^{2}}{5 R^{2}}, A_{2}^{\prime}=\frac{b^{\prime 2}-a^{\prime 2}}{5 R^{2}},\left(A_{1}, A_{2}, A_{1}^{\prime}, A_{2}^{\prime} \ll 1\right),
\end{gathered}
$$

$a, b, c=$ length of the semi axes of the triaxial body of mass $m_{1}$,

$a^{\prime}, b^{\prime}, c^{\prime}=$ length of the semi axes of the triaxial body of mass $m_{2}$,

$R=$ dimensional distance between the primaries,

$$
\begin{gathered}
r_{1}^{2}=(x+\mu)^{2}+y^{2}, r_{2}^{2}=(x+\mu-1)^{2}+y^{2}, \\
\mu=\frac{m_{2}}{m_{1}+m_{2}},
\end{gathered}
$$

$U=$ constant to be so chosen such that h (energy constant) will vanish at $L_{4}$ (libration point).

The coordinates of $L_{4}$ (libration) are

$$
\begin{gathered}
x_{L_{4}}=-\frac{1}{2}+\mu+\alpha_{1} A_{1}+\alpha_{1}^{\prime} A_{1}^{\prime}+\gamma_{1} A_{2}+\gamma_{1}^{\prime} A_{2}^{\prime}+\beta_{1} P+\beta_{1}^{\prime} P^{\prime}, \\
y_{L_{4}}=\frac{\sqrt{3}}{2}+\alpha_{2} A_{1}+\alpha_{2}^{\prime} A_{1}^{\prime}+\gamma_{2} A_{2}+\gamma_{2}^{\prime} A_{2}^{\prime}+\beta_{2} P+\beta_{2}^{\prime} P^{\prime},
\end{gathered}
$$


where $\alpha_{1}=-\frac{1}{2}, \alpha_{1}^{\prime}=\frac{1}{2}, \alpha_{2}=-\frac{1}{2 \sqrt{3}}, \alpha_{2}^{\prime}=-\frac{1}{2 \sqrt{3}}$,

$$
\begin{gathered}
\gamma_{1}=-\frac{7}{8}-\frac{1}{2 \mu}, \quad \gamma_{1}^{\prime}=\frac{7}{8}+\frac{1}{2(1-2 \mu)}, \quad \gamma_{2}=\frac{\sqrt{3}}{2}\left(\frac{5}{4}-\frac{1}{3 \mu}\right), \quad \gamma_{2}^{\prime}=\frac{\sqrt{3}}{2}\left(\frac{5}{4}-\frac{1}{3(1-\mu)}\right), \\
\beta_{1}=\frac{1}{3}, \quad \beta_{1}^{\prime}=-\frac{1}{3}, \quad \beta_{2}=-\frac{1}{3 \sqrt{3}}, \quad \beta_{2}^{\prime}=-\frac{1}{3 \sqrt{3}}
\end{gathered}
$$

and

$$
\begin{aligned}
U= & -\frac{1}{2}\left(3-\mu+\mu^{2}\right)-\frac{A_{1}}{4}\left(3-\mu+3 \mu^{2}\right)-\frac{A_{1}^{\prime}}{4}\left(5-5 \mu+3 \mu^{2}\right) \\
& -\frac{A_{2}}{8 \mu}\left(6 \mu+11 \mu^{2}-2 \mu^{3}-4\right)-\frac{A_{2}^{\prime}}{8(1-\mu)}\left(11+2 \mu+24 \mu^{2}-37 \mu^{3}\right) \\
& +\mu P+(1-\mu) P^{\prime} .
\end{aligned}
$$

Equations of motion can also be written as

$$
\begin{gathered}
\ddot{x}-n \dot{y}=W_{x}, \\
\ddot{y}+2 n \dot{x}=W_{y}
\end{gathered}
$$

where

$$
\begin{aligned}
W= & \frac{n^{2}}{2}\left(x^{2}+y^{2}\right)+(1-\mu)\left(\frac{1}{r_{1}}+\frac{A_{1}}{2 r_{1}^{3}}+\frac{3 A_{2} y^{2}}{2 r_{1}^{5}}-\frac{P}{r_{1}}\right) \\
& +\mu\left(\frac{1}{r_{2}}+\frac{A_{1}^{\prime}}{2 r_{2}^{3}}+\frac{3 A_{2}^{\prime} y^{2}}{2 r_{2}^{5}}-\frac{P^{\prime}}{r_{2}}\right)+U+h,
\end{aligned}
$$

The Jacobi integral is

$$
\begin{aligned}
C= & \frac{1}{2}\left(\dot{x}^{2}+\dot{y}^{2}\right)-\frac{n^{2}}{2}\left(x^{2}+y^{2}\right)-(1-\mu)\left(\frac{1}{r_{1}}+\frac{A_{1}}{2 r_{1}^{3}}+\frac{3 A_{2} y^{2}}{2 r_{1}^{5}}-\frac{P}{r_{1}}\right) \\
& -\mu\left(\frac{1}{r_{2}}+\frac{A_{1}^{\prime}}{2 r_{2}^{3}}+\frac{3 A_{2}^{\prime} y^{2}}{2 r_{2}^{5}}-\frac{P^{\prime}}{r_{2}}\right)-U \equiv h .
\end{aligned}
$$

\section{Normal and Tangent Variables}

We consider the system of generalized coordinates $Q=(x, y)^{\mathrm{T}}$. They depend upon the eight parameters $p=\left(\mu, h, A_{1}, A_{2}, A_{1}^{\prime}, A_{2}^{\prime}, P, P^{\prime}\right)^{\mathrm{T}}$. The corresponding differential equations are given by the system of Equation (2) with Jacobi integral given by (3). We consider the solutions of Equations (2) for which $C$ is zero. If we consider the solutions of Equation (2) given by (4) for some fixed parameters value $p$ then there may exist another solution given by (5) with another parameter value $p^{*}=\left(\mu^{*}, h^{*}, A_{1}^{*}, A_{2}^{*}, A_{1}^{* *}, A_{2}^{\prime *}, P^{*}, P^{\prime^{*}}\right)^{\mathrm{T}}$ close to $p$. We have

$$
\begin{aligned}
& x=x\left(\mu, h, A_{1}, A_{2}, A_{1}^{\prime}, A_{2}^{\prime}, P, P^{\prime}\right), \\
& y=y\left(\mu, h, A_{1}, A_{2}, A_{1}^{\prime}, A_{2}^{\prime}, P, P^{\prime}\right), \\
& \dot{x}=\dot{x}\left(\mu, h, A_{1}, A_{2}, A_{1}^{\prime}, A_{2}^{\prime}, P, P^{\prime}\right), \\
& \dot{y}=\dot{y}\left(\mu, h, A_{1}, A_{2}, A_{1}^{\prime}, A_{2}^{\prime}, P, P^{\prime}\right),
\end{aligned}
$$

and 


$$
\begin{aligned}
& x^{*}=x\left(\mu^{*}, h^{*}, A_{1}^{*}, A_{2}^{*}, A_{1}^{*}, A_{2}^{\prime *}, P^{*}, P^{\prime *}\right), \\
& y^{*}=y\left(\mu^{*}, h^{*}, A_{1}^{*}, A_{2}^{*}, A_{1}^{\prime *}, A_{2}^{\prime *}, P^{*}, P^{\prime *}\right), \\
& \dot{x}^{*}=\dot{x}\left(\mu^{*}, h^{*}, A_{1}^{*}, A_{2}^{*}, A_{1}^{*}, A_{2}^{\prime *}, P^{*}, P^{\prime *}\right), \\
& \dot{y}^{*}=\dot{y}\left(\mu^{*}, h^{*}, A_{1}^{*}, A_{2}^{*}, A_{1}^{\prime *}, A_{2}^{\prime *}, P^{*}, P^{\prime *}\right),
\end{aligned}
$$

Solution (5) will reduce to Solution (4) as $p^{*} \rightarrow p$.

Now we give the displacements

$$
\begin{gathered}
\Delta p=p^{*}-p \text { and } \xi=Q^{*}-Q,\left(\text { where } Q^{*}=\left(x^{*}, y^{*}\right)^{\mathrm{T}} \text { and } \xi=\left(\xi_{1}, \xi_{2}\right)^{\mathrm{T}}\right) \\
\text { i.e., } \Delta \mu=\mu^{*}-\mu, \Delta h=h^{*}-h, \Delta A_{1}=A_{1}^{*}-A_{1}, \Delta A_{2}=A_{2}^{*}-A_{2}, \\
\Delta A_{1}^{\prime}=A_{1}^{*}-A_{1}^{\prime}, \Delta A_{2}^{\prime}=A_{2}^{\prime *}-A_{2}^{\prime}, \Delta P=P^{*}-P, \Delta P^{\prime}=P^{*}-P^{\prime}
\end{gathered}
$$

and

$$
\xi_{1}=x^{*}-x, \quad \xi_{2}=y^{*}-y,
$$

We consider $\Delta p$ and $\xi$ as small quantities of the same order. Then we have the following variational equations:

$$
\begin{aligned}
& \ddot{\xi}_{1}=W_{x x} \xi_{1}+W_{x y} \xi_{2}+2 n \dot{\xi}_{2}+W_{x \mu} \Delta \mu+W_{x A_{1}} \Delta A_{1}+W_{x A_{2}} \Delta A_{2}+W_{x A_{1}} \Delta A_{1}^{\prime}+W_{x A_{2}^{\prime}} \Delta A_{2}^{\prime}+W_{x P} \Delta P+W_{x P^{\prime}} \Delta P^{\prime}, \\
& \ddot{\xi}_{2}=W_{x y} \xi_{1}+W_{y y} \xi_{2}-2 n \dot{\xi}_{1}+W_{y \mu} \Delta \mu+W_{y A_{1}} \Delta A_{1}+W_{y A_{2}} \Delta A_{2}+W_{y A_{1}^{\prime}} \Delta A_{1}^{\prime}+W_{y A_{2}^{\prime}} \Delta A_{2}^{\prime}+W_{y P} \Delta P+W_{y P^{\prime}} \Delta P^{\prime}
\end{aligned}
$$

with the integral constructed from Equation (3), retaining the first order terms only, we get

$$
C=\dot{x} \dot{\xi}_{1}+\dot{y} \dot{\xi}_{2}-W_{x} \xi_{1}-W_{y} \xi_{2}-W_{\mu} \Delta \mu-W_{h} \Delta h-W_{A_{1}} \Delta A_{1}-W_{A_{2}} \Delta A_{2}-W_{A_{1}^{\prime}} \Delta A_{1}^{\prime}-W_{A_{2}^{\prime}} \Delta A_{2}^{\prime}-W_{P} \Delta P-W_{P^{\prime}} \Delta P^{\prime} .
$$

The modulus of momentary velocity on the orbit is defined as $V(t)=|\dot{Q}(t)|=\sqrt{\dot{x}^{2}+\dot{y}^{2}}$. We assume that (5) is not corresponding to the equilibrium state, i.e., $V(t) \neq 0$ and we further assume that $V(t) \neq 0$ on the whole orbit. Therefore, $x$ and $y$ become the mobile co-ordinates. We will, now, use the mobile coordinate system to draw the periodic orbits by resolving one of the axis along the velocity vector $X=(\dot{x}, \dot{y})^{\mathrm{T}}$ and the other axis along the normal vector $Y=(-\dot{y}, \dot{x})^{\mathrm{T}}$.

In the new coordinate system, we consider the transition matrix $S$ as follows:

Consider the first column of $S$ as

$r(t)=\frac{Y(t)}{V(t)}=$ the unit vector which is normal to the orbit, i.e., it is orthogonal to the vector $s(t)$.

$s(t)=\frac{X(t)}{V(t)}=$ the unit vector which is tangent to the orbit and is the last column of the matrix $S$.

So, we have $S=\{r, s\}, \operatorname{dim}(r)=2 \times 1, \operatorname{dim}(s)=2 \times 1$, so that $\operatorname{dim}(s)=2 \times 2$.

It can be easily verified that

$$
s^{\mathrm{T}} s=1, r^{\mathrm{T}} s=0 \text { and } S^{-1} s=e=(0,1)^{\mathrm{T}}
$$

It may be noted that,

$$
r=\frac{1}{V(t)}\left(\begin{array}{c}
-\dot{y} \\
\dot{x}
\end{array}\right), s=\frac{1}{V(t)}\left(\begin{array}{c}
\dot{x} \\
\dot{y}
\end{array}\right), S=\frac{1}{V(t)}\left(\begin{array}{rr}
-\dot{y} & \dot{x} \\
\dot{x} & \dot{y}
\end{array}\right) \text { and } S^{-1}=S^{T}=\frac{1}{V(t)}\left(\begin{array}{rr}
-\dot{y} & \dot{x} \\
\dot{x} & \dot{y}
\end{array}\right) .
$$

Now, we may further define

$$
\begin{aligned}
& r^{*}=r^{\mathrm{T}}=\frac{1}{V(t)}\left(\begin{array}{ll}
-\dot{y} \dot{x}
\end{array}\right), \text { the first line of } S^{-1}, \\
& s^{*}=s^{\mathrm{T}}=\frac{1}{V(t)}\left(\begin{array}{ll}
\dot{x} & \dot{y}
\end{array}\right), \text { the last line of } S^{-1} .
\end{aligned}
$$


We write $\alpha$, the vector of local coordinates, in the new coordinate system as follows:

$$
\alpha=\left(\begin{array}{l}
N \\
M
\end{array}\right), \operatorname{dim}(N)=1 \times 1 \text { and } \operatorname{dim}(M)=1 \times 1,
$$

where $N$ is displacement along the normal to the orbit and $M$ is displacement along the tangent to the orbit.

Then, the new coordinates are given by

$$
\begin{gathered}
\xi=S \alpha=\left(\begin{array}{ll}
r & s
\end{array}\right)\left(\begin{array}{l}
N \\
M
\end{array}\right)=r N+s M \\
\alpha=S^{-1} \xi, \quad N=r^{*} \xi, \quad M=s^{*} \xi, \\
\dot{\xi}=\dot{S} \alpha+S \dot{\alpha}=\dot{r} N+r \dot{N}+\dot{S} M+s \dot{M}
\end{gathered}
$$

Substituting these values into the integral (7a), we have

$$
\begin{aligned}
C= & \frac{2 W}{V^{2}}(\dot{M} V-M \dot{V})+\frac{1}{V}\left(W_{x} \dot{y}-W_{y} \dot{x}+\ddot{x} \dot{y}-\ddot{x} \ddot{y}\right) N-W_{\mu} \Delta \mu \\
& -W_{h} \Delta h-W_{A_{1}} \Delta A_{1}-W_{A_{2}} \Delta A_{2}-W_{A_{1}} \Delta A_{1}^{\prime} \\
& -W_{A_{2}} \Delta A_{2}^{\prime}-W_{P} \Delta P-W_{P^{\prime}} \Delta P^{\prime} \equiv 0 .
\end{aligned}
$$

Equation (10) can be solved for $\dot{M}$ as

$$
\begin{aligned}
\dot{M}= & \frac{M \dot{V}}{V}-\frac{1}{2 W}\left(W_{x} \dot{y}-W_{y} \dot{x}+\ddot{x} \dot{y}-\ddot{x} \ddot{y}\right) N \\
& +\frac{1}{V}\left(W_{\mu} \Delta \mu+W_{h} \Delta h+W_{A_{1}} \Delta A_{1}+W_{A_{2}} \Delta A_{2}+W_{A_{1}^{\prime}} \Delta A_{1}^{\prime}+W_{A^{\prime}} \Delta A_{2}^{\prime}+W_{P} \Delta P+W_{P^{\prime}} \Delta P^{\prime}\right) .
\end{aligned}
$$

Equations of motion (2) for the new coordinates are

$$
S \ddot{\alpha}=\ddot{\xi}-2 \dot{S} \dot{\alpha}-\ddot{S} \alpha \text { or } \ddot{\alpha}=S^{-1}\left(F_{N} N+F_{\dot{N}} \dot{N}+F_{\Delta p} \Delta p+\frac{\ddot{V}}{V} M s\right) .
$$

where $F_{\Delta p}=\left(\begin{array}{llllllll}F_{\mu}^{1} & F_{h}^{2} & F_{A_{1}}^{3} & F_{A_{2}}^{4} & F_{A_{1}^{\prime}}^{5} & F_{A_{2}^{\prime}}^{6} & F_{P}^{7} & F_{P^{\prime}}^{8}\end{array}\right)$,

$$
\begin{gathered}
F_{\alpha_{l}}^{l}=\left(\begin{array}{c}
W_{x \alpha_{l}}+\frac{W_{\alpha_{l}}}{W}\left(n \dot{y}+\frac{\dot{V}}{V} \dot{x}-\ddot{x}\right) \\
W_{y \alpha_{l}}+\frac{W_{\alpha_{l}}}{W}\left(-n \dot{x}+\frac{\dot{V}}{V} \dot{y}-\ddot{y}\right)
\end{array}\right), \quad l=1 \text { to } 8, \\
\alpha_{1}=\mu, \alpha_{2}=h, \alpha_{3}=A_{1}, \alpha_{4}=A_{2}, \alpha_{5}=A_{1}^{\prime}, \alpha_{6}=A_{2}^{\prime}, \alpha_{7}=P, \alpha_{8}=P^{\prime},
\end{gathered}
$$

$W_{x \alpha_{2}}=0$ and $W_{y \alpha_{2}}=0$ and $F_{N}$ and $F_{\dot{N}}$ are the same as in our papers [1]-[3].

Since $\alpha=\left(\begin{array}{l}N \\ M\end{array}\right)$, therefore the equations of motion in normal and tangent co-ordinates can be written as

$$
\ddot{N}=r^{*}\left(F_{N} N+F_{\dot{N}} \dot{N}+F_{\Delta P} \Delta P\right)
$$

and

$$
\ddot{M}=\frac{\ddot{V}}{V} M+s^{*}\left(F_{N} N+F_{\dot{N}} \dot{N}+F_{\Delta P} \Delta P\right) .
$$

$\ddot{N}$ can also be written as 


$$
\begin{aligned}
\ddot{N}= & \frac{1}{V^{2}}\left(W_{x x} \dot{y}^{2}-2 W_{x y} \dot{x} \dot{y}+W_{y y} \dot{x}^{2}+\frac{1}{W}\left(-W_{x} \dot{y}+W_{y} \dot{x}\right)\left(-n V^{2}+\dddot{x} \dot{y}-\dot{x} \ddot{y}\right)\right. \\
& \left.-2 n(\ddot{x} \dot{y}-\dot{x} \ddot{y})-\frac{1}{W}(\dddot{x} \dot{y}-\dot{x} \ddot{y})\left(-n V^{2}+\ddot{x} \dot{y}-\dot{x} \ddot{y}\right)+\ddot{x}^{2}+\ddot{y}^{2}-\dot{V}^{2}\right) N \\
& +\left(\frac{1}{V}\left(-W_{x \mu} \dot{y}+W_{y \mu} \dot{x}+\frac{W_{\mu}}{W}\left(-n V^{2}+\dddot{x} \dot{y}-\dot{x} \ddot{y}\right)\right) \Delta \mu+\sum_{i=1}^{2}\left(-W_{x A_{i}} \dot{y}+W_{y A_{i}} \dot{x}+\frac{W_{A_{i}}}{W}\left(-n V^{2}+\dddot{x} \dot{y}-\dot{x} \ddot{y}\right)\right) \Delta A_{i}\right. \\
& +\sum_{i=1}^{2}\left(-W_{x A^{\prime}} \dot{y}+W_{y A_{i}^{\prime}} \dot{x}+\frac{W_{A^{\prime}}}{W}\left(-n V^{2}+\dddot{x} \dot{y}-\dot{x} \ddot{y}\right)\right) \Delta A_{i}^{\prime}+\left(-W_{x P} \dot{y}+W_{y P} \dot{x}+\frac{W_{P}}{W}\left(-n V^{2}+\dddot{x} \dot{y}-\ddot{x} \ddot{y}\right)\right) \Delta P \\
& \left.+\left(-W_{x P^{\prime}} \dot{y}+W_{y P^{\prime}} \dot{x}+\frac{W_{P^{\prime}}}{W}\left(-n V^{2}+\dddot{x} \dot{y}-\ddot{x} \ddot{y}\right)\right) \Delta P^{\prime}+\frac{W_{h}}{W}\left(-n V^{2}+\dddot{x} \dot{y}-\ddot{x} \ddot{y}\right) \Delta h\right) .
\end{aligned}
$$

Thus, we have derived the equation in $\ddot{N}$ which possesses the remarkable property that the normal coordinate $(N)$ is independent of the tangent coordinate $(M)$. Moreover, instead of the differential equation of the second order (13), we can use the first order differential Equation (11). If the investigated motion (4) is periodic, then the matrix $S(t)$ can be taken as periodic and Equations (12) and (13) will have the periodic coefficients at $\Delta p \equiv 0$.

\section{Periodic Orbits}

For determining the periodic orbits, the required equations of motion and the variational equations are given as:

$$
\begin{gathered}
\ddot{x}=2 n \dot{y}+W_{x} \\
\ddot{y}=-2 n \dot{x}+W_{y} \\
\dot{Z}_{j}=\left(\begin{array}{cc}
0 & I_{J} \\
r^{*} F_{N} & r^{*} F_{\dot{N}}
\end{array}\right) Z_{j}, Z_{j}(0)=e_{j},(j=1, \cdots, 2 J) \\
\dot{\mu}_{j}=\frac{\dot{V}}{V} \mu_{j}-\frac{V}{2 W} g_{v} Z_{j}, \mu_{j}(0)=0 \\
\dot{v}_{\Delta P_{k}}=\left(\begin{array}{cc}
0 & I_{J} \\
r^{*} F_{N} & r^{*} F_{\dot{N}}
\end{array}\right) v_{\Delta P_{k}}+\left(\begin{array}{c}
0 \\
r^{*} F_{\Delta P_{k}}
\end{array}\right), v_{\Delta P_{k}}(0)=0,(k=1, \cdots, K) \\
\dot{\mu}_{\Delta P_{k}}=\frac{\dot{V}_{V}}{V} \mu_{\Delta P_{k}}-\frac{V}{2 W}\left(g_{v} v_{\Delta P_{k}}+g_{P_{k}}\right), \mu_{\Delta P_{k}}(0)=0 .
\end{gathered}
$$

where $Z(t)$ is the matrix of solutions of a homogeneous system with initial condition $Z(0)=I_{2 J}$ and $v_{\Delta P_{k}}(t)=a$ particular solution of the equations with zero initial conditions, i.e., $v_{\Delta P_{k}}(0)=0$. The row-vector $\mu(t)$ and $\mu_{\Delta P_{k}}(t)$ are the solutions of Cauchy problem (iv) with (vi) of (14). The order of the above system is thirty-four.

So, for finding the new periodic motion it is necessary to integrate the system (14) of the differential equations from $t=0$ and $t=T$. In the formulae (i) to (vi) of (14), it may be noted that $I_{2 J}=\left(e_{1} \ldots e_{2 J}\right), \mathrm{Z}=\left(Z_{1} \ldots Z_{2 J}\right), \mu$ $=\left(\mu_{1}, \ldots, \mu_{2 J}\right)$ and the initial conditions $x(0), y(0), \dot{x}(0)$ and $\dot{y}(0)$ are known.

After solving the above equations of motion (i) and (ii), the variational Equations (iii)-(vi) of (14) and applying the predictor-corrector method, we have determined the periodic orbits.

We have drawn the periodic orbits for the following:

1) for fixed $\mu=0.001, A_{1}=0.0, A_{2}=0.0, A_{1}^{\prime}=0.001, A_{2}^{\prime}=0.0, P=0.0$ and $P^{\prime}=0.0$ (Figure 2);

2) for fixed $\mu=0.001, A_{1}=0.001, A_{2}=0.0, A_{1}^{\prime}=0.001, A_{2}^{\prime}=0.0, P=0.0001$ and $P^{\prime}=0.00$ (Figure 3);

3 ) for fixed $\mu=0.001, A_{1}=0.001, A_{2}=0.001, A_{1}^{\prime}=0.0, A_{2}^{\prime}=0.0, P=0.0$ and $P^{\prime}=0.0001$ (Figure 4);

4) for fixed $\mu=0.001, A_{1}=0.001, A_{2}=0.001, A_{1}^{\prime}=0.001, A_{2}^{\prime}=0.001, P=0.0001$ and $P^{\prime}=0.0001$ (Figure 5);

5) for fixed $\mu=0.001, A_{1}=0.002, A_{2}=0.003, A_{1}^{\prime}=0.004, A_{2}^{\prime}=0.005, P=0.001$ and $P^{\prime}=0.001$ (Figure 6). 
y
1) $h=0.05$
2) $h=0.10$
3) $h=0.15$
4) $h=0.20$
5) $h=0.3258$

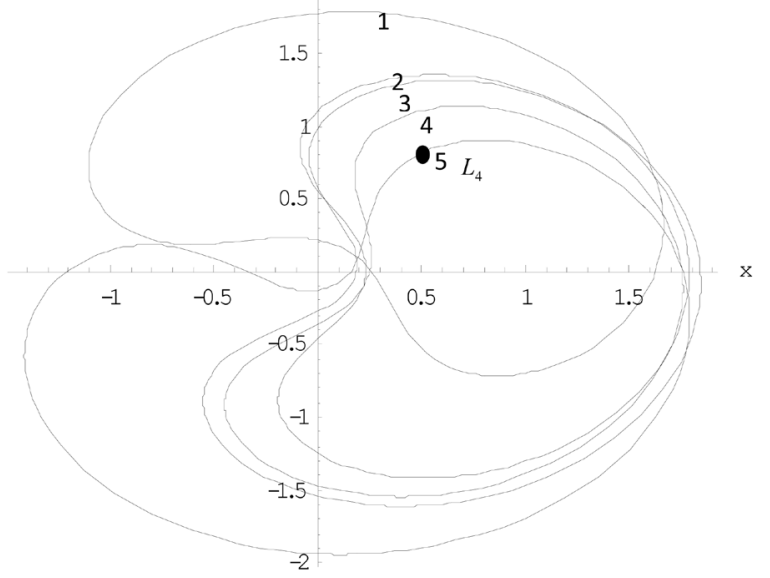

Figure 2. Periodic orbits when $\mu=0.001, A_{1}=0.0, A_{2}=0.0, A_{1}^{\prime}=0.001, A_{2}^{\prime}=0.0, P=$ 0.0 and $P^{\prime}=0.0$.

1) $h=0.15$
2) $h=0.20$
3) $h=0.23$
4) $h=0.28$
5) $h=0.31205$

y

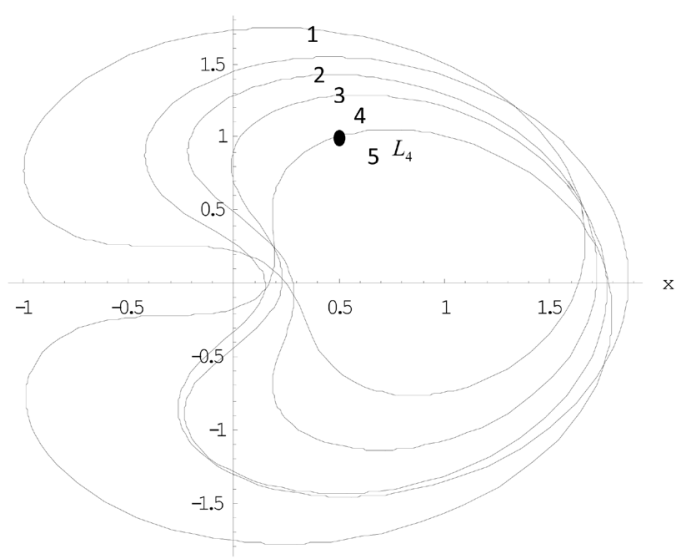

Figure 3. Periodic orbits when $\mu=0.001, A_{1}=0.001, A_{2}=0.0, A_{1}^{\prime}=0.001, A_{2}^{\prime}=0.0, P=$ 0.0001 and $P^{\prime}=0.0$.
1) $h=0.25$
2) $h=0.20$
3) $h=0.15$
4) $h=0.10$
5) $h=0.0895$

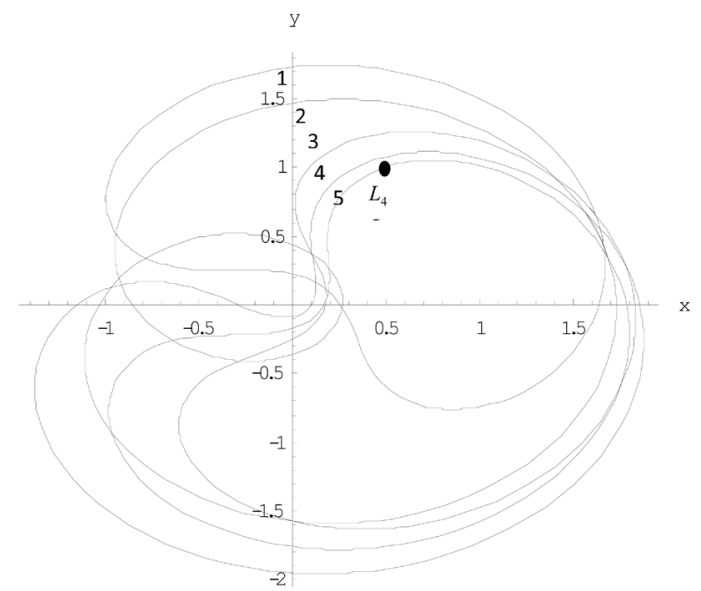

Figure 4. Periodic orbits when $\mu=0.001, A_{1}=0.001, A_{2}=0.001, A_{1}^{\prime}=0.0, A_{2}^{\prime}=0.0, P=$ 0.00 and $P^{\prime}=0.0001$. 
1) $h=0.28$

2) $h=0.23$

3) $h=0.18$

4) $h=0.12$

5) $h=0.09225$

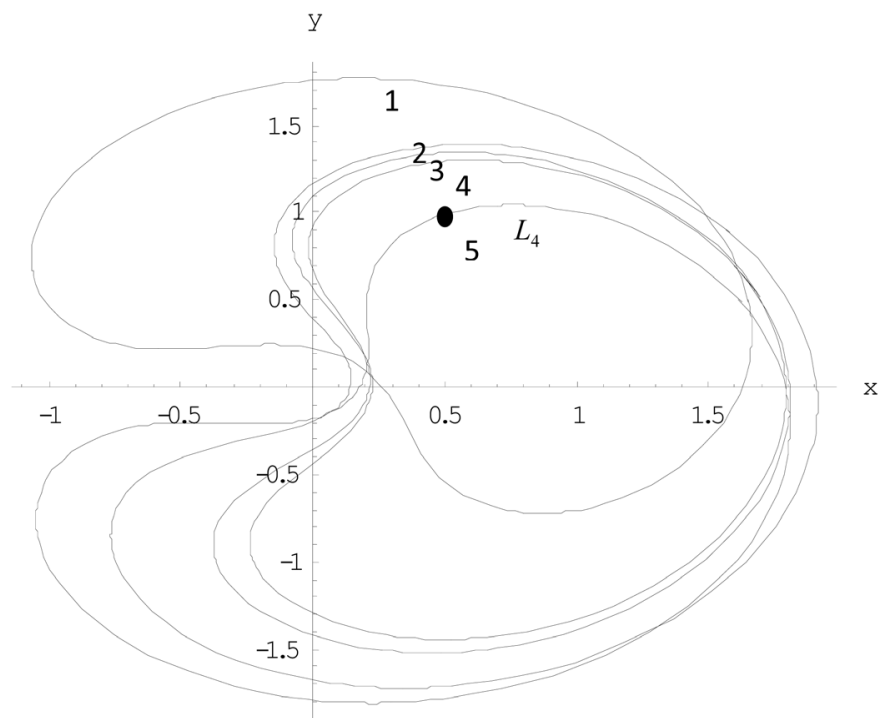

Figure 5. Periodic orbits when $\mu=0.01, A_{1}=0.001, A_{2}=0.001, A_{1}^{\prime}=0.001, A_{2}^{\prime}=0.001, P$ $=0.0001$ and $P^{\prime}=0.0001$.
1) $h=0.30$
2) $h=0.22$
3) $h=0.16$
4) $h=0.15$
5) $h=0.10215$

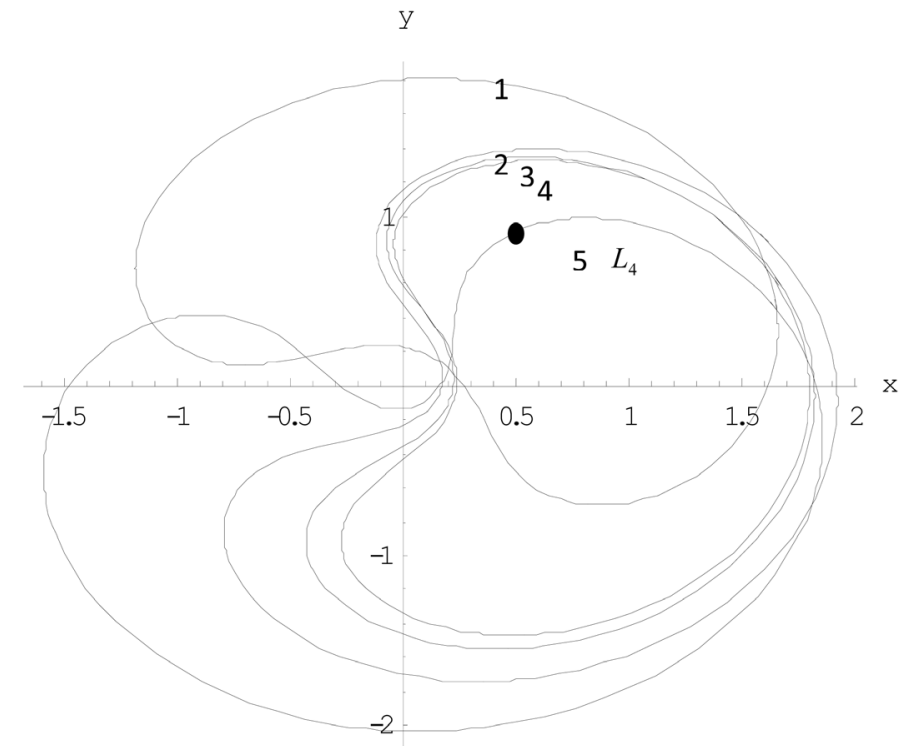

Figure 6. Periodic orbits when $\mu=0.003, A_{1}=0.002, A_{2}=0.003, A_{1}^{\prime}=0.004, A_{2}^{\prime}=0.005, P$ $=0.001$ and $P^{\prime}=0.001$.

In each figure, we have drawn 5 periodic orbits corresponding to different values of $h$. These orbits have been numbered 1, 2, 3, 4 and 5 corresponding to different values of $h$.

The above analysis is summed up in Table 1. By taking both the primaries as radiating triaxial rigid bodies, the difference in the behavior of the values of $h$ is obvious.

\section{Conclusions}

Karimov and Sokolsky [11] have studied periodic orbits in the restricted three body problem by giving the displacements along the normal and the tangent to the orbit at the mobile co-ordinates. They have taken both the primaries as point masses while in this paper besides taking both the primaries as triaxial rigid bodies, we have also taken both the primaries as source of radiation pressure as well. In this paper, we have again determined 
Table 1. The summary analysis of the periodic orbits.

\begin{tabular}{cccccc}
\hline$\mu=0.001$ & Oblate body Figure 2 & Triaxial body Figure 3 & Triaxial body Figure 4 & Triaxial body Figure 5 & Triaxial body Figure 6 \\
\hline & $A_{1}=0.0$ & $A_{1}=0.001$ & $A_{1}=0.001$ & $A_{1}=0.001$ & $A_{1}=0.002$ \\
$\begin{array}{c}\text { Values of } \\
\text { Energy }\end{array}$ & $A_{1}^{\prime}=0.001$ & $A_{1}^{\prime}=0.001$ & $A_{1}^{\prime}=0.0$ & $A_{1}^{\prime}=0.001$ & $A_{1}^{\prime}=0.003$ \\
$\begin{array}{c}\text { Constant } \\
h\end{array}$ & $A_{2}=0.0$ & $A_{2}=0.0$ & $A_{2}=0.001$ & $A_{2}=0.001$ & $A_{2}=0.004$ \\
& $A_{2}^{\prime}=0.0$ & $A_{2}^{\prime}=0.0$ & $A_{2}^{\prime}=0.0$ & $A_{2}^{\prime}=0.001$ & $A_{2}^{\prime}=0.005$ \\
$P_{1}=0.0$ & $P^{\prime}=0.0001$ & $P^{\prime}=0.0$ & $P^{\prime}=0.0001$ & $P=0.001$ \\
$h_{2}$ & $P^{\prime}=0.0$ & $P^{\prime}=0.0$ & $P^{\prime}=0.0001$ & $P^{\prime}=0.0001$ & $P^{\prime}=0.001$ \\
$h_{3}$ & 0.05 & 0.15 & 0.25 & 0.28 & 0.30 \\
$h_{4}$ & 0.10 & 0.20 & 0.20 & 0.23 & 0.22 \\
$h_{5}$ & 0.15 & 0.23 & 0.15 & 0.18 & 0.16 \\
\hline
\end{tabular}

five periodic orbits in a family for fixed value of the mass parameter $\mu$, the triaxial parameters $A_{1}, A_{2}, A_{1}^{\prime}, A_{2}^{\prime}$ and the radiation parameters $P$ and $P^{\prime}$ with varying energy constant $h$.

We have observed the following effects on the periodic orbits and on the energy constant $h$ due to triaxial rigid bodies and radiation pressure if we compare it with the results of Karimov and Sokolsky [11] and our papers [1]-[3].

1) The energy constant $h$ increases in a family (for Figure 2 and Figure 3 ) then it decreases (for Figures 4-6) for fixed trixial parameters $A_{1}, A_{2}, A_{1}^{\prime}, A_{2}^{\prime}$ and radiation parameters $P$ and $P^{\prime}$.

2) As we increase the radiation parameters $P$ and $P^{\prime}$, the energy constant $h$ increases whereas the periodic orbits shrink a little.

3) The periodic orbits go away from the libration point $L_{4}$ as we increase triaxial parameters $A_{1}, A_{2}, A_{1}^{\prime}, A_{2}^{\prime}$ and radiation parameters $P$ and $P^{\prime}$ whereas energy constant $h$ decrease.

We have investigated the family up to the member which touches the point $L_{4}$. It is observed that the families of periodic orbits in Karimov and Sokolsky [11] terminate at both the triangular equilibrium points simultaneously, while in our case these families are non-symmetrical, so they may continue.

\section{Acknowledgements}

We are thankful to the Centre for Fundamental Research in Space Dynamics and Celestial Mechanics (CFRSC), Delhi and the Deanship of Scientific Research, College of Science in Zulfi, Majmaah University, KSA for providing all the research facilities in the completion of this research work.

\section{References}

[1] Mittal, A., Aggarwal, R. and Bhatnagar, K.B. (2011) Periodic Orbits around $L_{4}$ in the Photogravitational Restricted Problem with Oblate Primaries. WSEAS 6th International Conference Proceedings on Optics Astrophysics and Astrology, Article ID: 650927.

[2] Mittal, A., Iqbal, A. and Bhatnagar, K.B. (2008) Periodic Orbits Generated by Lagrangian Solutions of the Restricted Three-Body Problem When One of the Primaries Is an Oblate Body. Astrophysics and Space Science, 319, 63-73. http://dx.doi.org/10.1007/s10509-008-9942-0

[3] Mittal, A., Iqbal, A. and Bhatnagar, K.B. (2009) Periodic Orbits in the Photogravitational Restricted Problem with the Smaller Primary an Oblate Body. Astrophysics and Space Science, 323, 65-73. http://dx.doi.org/10.1007/s10509-009-0038-2

[4] Charlier, C.L. (1899) Die Mechanik des Himmels. Walter de Gryter and Co., Berlin and Leipzig.

[5] Plummer, H.C. (1901) On Periodic Orbits in the Neighborhood of Centres of Liberation. Monthly Notices of the Royal Astronomical Society, 62, 6-17. http://dx.doi.org/10.1093/mnras/62.1.6

[6] Riabov, U.A. (1952) Preliminary Orbits Trojan Asteroids. Soviet Astronomy, 29, 5. 
[7] Szebehely, V. (1967) Theory of Orbits: The Restricted Problem of Three Bodies. Academic Press, New York.

[8] Deprit, A. and Henrard, J. (1968) Advances in Astronomy and Astrophysics. Academic Press, New York, London.

[9] Markeev, A.P. and Sokolsky, A.G. (1975) Investigation of Periodic Motions near the Lagrangian Solutions of Restricted Three-Body Problem. Publ. Inst. of Appl. Math. Acad. Sci., Moscow.

[10] Hadjidemetriou, J.D. (1984) Periodic Orbits. Celestial Mechanics, 34, 379-393. http://dx.doi.org/10.1007/BF01235816

[11] Karimov, S.R. and Sokolsky, A.G. (1989) Periodic Motions Generated by Lagrangian Solutions of the Circular Restricted Three-Body Problem. Celestial Mechanics and Dynamical Astronomy, 46, 335-381. http://dx.doi.org/10.1007/BF00051487

[12] Taqvi, Z.A.A.R. and Iqbal, A. (2006) Non-Linear Stability of $L_{4}$ in the Restricted Three-Body Problem for Radiated Axes Symmetric Primaries with Resonances. Bulletin of Astronomical Society of India, 35, 1-29.

[13] Abouelmagd, E.I., Alhothuali, M.S., Guirao, J.L.G. and Malaikah, H.M. (2015) Periodic and Secular Solutions in the Restricted Three-Body Problem under the Effect of Zonal Harmonic Parameters. Applied Mathematics \& Information Sciences, 9, 1659-1669.

[14] Perdios, E.A., Kalantonis, V.S., Perdiou, A.E. and Nikaki, A.A. (2015) Equilibrium Points and Related Periodic Motions in the Restricted Three-Body Problem with Angular Velocity and Radiation Effects. Advances in Astronomy, 2015, 1-21.

[15] Jain, M. and Aggarwal, R. (2015) A Study of Non-Collinear Libration Points in Restricted Three-Body Problem with Stokes Drag Effect When Smaller Primary Is an Oblate Spheroid. Astrophysics and Space Science, 358, 1-8. 